\title{
Comisión de Estudio sobre Toxemia Gravídica
}

Comentario sobre el trabajo: "Observaciones Sobre los Tratamientos de la Eclampsia" presentado por el doctor Silva-Mojica en la sesión ordinaria de la Sociedad del día 10 de diciembre de 1953.

Según consta en el acta de la sesión de este día, dicho trabajo fue comentado y discutido extensamente. Hicieron observaciones muy interesantes al respecto los doctores Jaime Pedraza, Jaime Afanador, Miguel A. Fernández, Mario Sanz Aráoz, Luis Salazar Dávila, Eugenio Pérez, Agustín Pachón, Camilo Casas y otros varios. Tanto el doctor Afanador como el doctor Fernández Bastidas se manifestaron decididamente partidarios de insistir en el tratamiento médico de la toxemia gravídica, pero efectuada en una forma racional y lógica: dieta apropiada, recinto especial para aislar a las toxémicas, personal de enfermeras con entrenamiento especial para estos tratamientos, y necesidad de algunos exámenes de laboratorio, como pruebas de funcionamiento renal y dosificación de uricemia.

Los doctores Salazar-Dávila y Sanz-Aráoz pusieron de presente que en el Instituto de Protección Materna e Infantil estaban ensayando un nuevo tratamiento para la toxemia gravídica con el "cocktail lítico", de la escuela francesa a base de fenergán, dolosal y largactil disuelto en solución dextrosada y aplicado por vía intravenosa. Consideran que los resultados aún no son efectivos por estar en vía de experimentación.

Otros colegąs, se manifestaron decididamente partidarios del tratamiento quirúrgico, sobre todo en casos de fracaso de inducción tanto en la preeclampsia grave, como en la eclampsia convulsiva declarada.

El doctor Durán-Forero manifestó que se debería ensayar en la toxemia gravídica algunos tratamientos que se están practicando en Norteamérica a base de antibióticos: terramicina y penicilina.

El doctor Silva-Mojica insistió en adoptar las bases por él presentadas en su trabajo para el tratamiento de la toxemia-pero en todo caso cree necesario una revisión detenida de nuestros sistemas de tratamiento médico. En esta idea lo secundaron el doctor Pérez-Gómez, el doctor Fernández-Bastidas y el doctor Afanador. En tal sentido se nombró una comisión destinada a iniciar, desde el $1^{\text {0 }}$ de enero de 1954, un detenido estudio sobre la toxemia gravídica en las diferentes clínicas y hospitales de Bogotá.

\section{Los detalles de la discusión suscitada esa noche fueron los siguientes:}

Dr. PEDRAZA.-Considera las observaciones del doctor Silva como el mejor resumen que sobre este tema puede hacerse. En cuanto a tratamientos mé- 
dicos que son muy variados, opina que el cocktail lítico con Largactyl, que se está ensayando, es de poca utilidad como lo comprobó en un caso que tuvo en la Clínica Primero de Mayo.

Dr. SALAZAR.-El llamado cocktail lítico está en vía de ensayo. Ha habido resultados buenos y nulos pero todavía no se puede concluir sobre su efectividad.

Dr. AFANADOR.-Lo que dice el doctor Silva en sus observaciones es lo que se observa comúnmente. Ante la pre-eclampsia no se puede permitir que se llegue a las convulsiones. Con una cifra de tensión arterial máxima de 180, se debe intervenir quirúrgicamente. Los norteamericanos e ingleses contraindican, por razones estadísticas el tratamiento quirúrgico en la eclampsia. Es de opinión que lo que sucede entre nosotros, y que determina muchas veces el fracaso del tratamiento médico, es que éste no se hace bien, con aislamiento y absoluto reposo de la enferma, etc., o que muy frecuentemente. se varía de sistemas, sin insistir en uno solo, sino usando varios a la vez e indiscriminadamente. Otra causa del fracaso es el hecho de que muchas veces no se diagnostica la enfermedad renal y si a ésta se agrega la toxemia, los resultados del tratamiento médico tienen que ser nulos. Deben delimitarse claramente la nefropatía pura y la toxemia gravídica para poder proceder en consecuencia.

Dr. SANZ.-Está de acuerdo con los conceptos del doctor Afanador en cuanto a no permitir que se presente la eclampsia convulsiva. Respecto del tratamiento con Largarctyl-Dolosal, en contraposición al caso del doctor Pedraza, refiere otro que trató en el Instituto de Protección Materno-infantil con las mismas drogas, en el cual la T. A. bajó en dos horas de 200 x 100 a 120 x 80 . Suspendida la aplicación se presentaron nuevas alzas de la T. A. que cedieron a nuevas aplicaciones. Cree que no pueden compararse las estadísticas de la Clinica Primero de Mayo con las del Instituto de Protección Materno-infantil porque en éste es mayor la incidencia de las toxemias y en aquélla las pacientes tienen un mejor control prenatal. El tratamiento ideal debe ser causal y es de esperar que se llegue a dominar completamente las toxemias por medio de la mejora de los servicios y de las condiciones físicas y culturales de las gestantes.

Dr. SILVA.-No es posible establecer resultados precisos del tratamiento médico, tan favorables como los del quirúrgico, y por eso se muestra partidario de este último.

Dr. FERNANDEZ.-Lo más importante es que se trate de hacer diagnóstico preciso de toxemia con exclusión de nefropatía, para no fracasar en el tratamiento. En un trabajo suyo, publicado en la Revista de la Sociedad, aparece una clasificación de las toxemias en que se revisan muchos conceptos que se nos enseñaron en la Facultad. Es indispensable practicar con la ayuda del laboratorio pruebas de función renal para hacer la diferencia enunciada, pero desgraciadamente a veces se tropieza con el costo alto de las pruebas de laboratorio. Se muestra partidario del tratamiento médico bien dirigido, con buen aislamiento, dietas bien equilibradas en cloruro de sodio y calorías, etc. Esto natu- 
ralmente es difícil y la Sociedad debería insinuar a las clínicas el uso de sistemas dietéticos convenientes. El régimen declorurado absoluto está contraindicado. A veces el solo régimen alimenticio bien dirigido es tratamiento suficiente.

En los métodos de tratamiento médico hay un verdadero caos: unos usan el sulfato de magnesia intravenoso y otros intramuscular con variaciones caprichosas en los períodos de aplicación y por otra parte nuestro individualismo impide sacar conclusiones de los muchos tratamientos empleados. Sería aconsejable standarizar un tipo de tratamiento y un régimen dietético y confrontar luego los resultados.

Dr. DURAN.-Sería difícil esa standarización porque todos los tratamientos están siendo revisados constantemente y las opiniones son diversas. Así por ejemplo, en algunos hospitales de Estados Unidos se dice que el sulfato de magnesia no da ningún resultado.

Dr. FERNANDEZ.-El sulfato de magnesia ha demostrado su buena acción espasmolítica y sedante, tan necesaria en las toxemias, de modo que su uso no puede relegarse. En nuestro medio, como dice el doctor Afanador, el tratamiento médico fracasa por incompleto y desordenado.

Dr. DURAN.--En la pasada Convención el doctor Salamanca se refirió a la raquianestesia continua como tratamiento médico de la toxemia. ¿Qué opiniones hay al respecto?

Dr. SILVA.-Tuvo ocasión de observar un caso del doctor Salamanca en que a pesar del citado tratamiento, hubo necesidad de operar.

Dr. FERNANDEZ.-Algunos autores americanos usan también la raquianestesia continua con buenos resultados.

Dr. SILVA.-Es un hecho que no hay continuidad en los tratamientos, pero podría ser mejor usar varios que se complementen y refuercen. Por ejemplo, el sulfato de magnesia y la dextrosa se refuerzan a veces con el Veratrum Viridae, con buenos resultados. Los profesores Acosta y Mojica empezaron a usar este último en nuestro medio hace mucho tiempo, aún antes que en los Estados Unidos, donde ahora está de moda.

Es de lamentar que en la Clínica Primero de Mayo se pierda el precioso material de investigación por fallas de estadística. Debe formarse un grupo de médicos que estudie los casos de toxemias en forma organizada y pedir al gerente del ICSS que se elabore un buen formato de historia clínica.

Dr. FERNANDEZ.-En la Clínica Primero de mayo existió una comisión de records qué quiso organizar la estadística, pero para ello eran necesarias buenas historias clínicas y al efecto se intentó modificar el formato existente, pero el doctor Vergara, entonces médico jefe del ICSS manifestó que a esa institución no le interesaba el control científico sino el contable y que para eso bastaba el cartón existente. También se organizaron reuniones científicas periódicas, pero todo se acabó por consunción pues nadie asistía. Está de acuerdo con 
el doctor Silva, pero cree que ese estudio estadístico debemos hacerlo nosotros, sin esperar colaboración del ICSS, así como la elaboración de un formato de historia clínica.

Dr. SALAZAR.-Se refiere de nuevo al caso del doctor Pedraza, tratado con el cocktail lítico y dice que probablemente se trataba de una nefrópata, porque la taza de albuminuria era de 36 grs. por mil. En cuanto a la variedad de tratamientos de la toxemia, cita el ejemplo de hospitales de Cincinatti donde usan el sulfato de magnesia al 50\% intramuscular!

Dr. PACHON.-No hay tratamiento específico de la toxemia; todos los tratamientos son sintomáticos. El sulfato de magnesia, la dextrosa, el fenobarbital, etc., se usan de una y otra manera. Ultimamente se está usando nuevamente el Veratrum Viridae. Fuera de algunos ensayos con otras drogas, todos usan lo mismo. Es de opinión que el tratamiento quirúrgico sí tiene razón de ser.

Dr. AFANADOR.-En discusiones médicas debe argumentarse a base de estadísticas demostrativas y no refiriéndose a experiencias personales solamente.

Dr. SILVA.-No ve por qué no deba hablarse de experiencias personales.

Dr. AFANADOR.-Porque esas experiencias, por valiosas que sean, no pueden dar normas.

Dr. CASAS.-En esta discusión se trata de toxicosis y el doctor Silva hace una magnífica síntesis sobre tratamiento de ellas, así: tratamiento quirúrgico ante la inminencia de eclampsia y en esta última ante la inminencia de coma. En síntesis, en la preeclampsia, evitar la eclampsia y en la eclampsia evitar el coma.

Dr. AFANADOR.--En la pre-eclampsia grave el tratamiento es quirúrgico pero la discusión está en la eclampsia en la que el tratamiento médico parece mejor.

Dr. NAVAS HERNANDO.-En el tratamiento quirúrgico de la eclampsia se tiene frecuentemente como factor de indicación operatoria la vida del feto, que puede perecer in-útero en el curso de un ataque, por anoxia fetal consecutiva a la apnea materna.

Dr. AFANADOR---Efectivamente, el feto está intoxicado y no resiste la anoxia. De ahí que el índice de mortinatalidad en el tratamiento quirúrgico de la eclampsia sea alto.

Dr. SILVA.-El doctor Plata Rueda observa que el hijo de la eclámptica tiene ahora mejor pronóstico por las mejores atenciones que recibe en la salacuna.

Dr. PEREZ.-Como resumen provechoso de todo lo que se ha dicho debe ponerse en práctica lo propuesto por el doctor Silva en formar una comisión que estudie los casos de toxemia que se presenten en todas las clínicas y hospitales de la ciudad y a la que se entreguen las historias de todos los.casos. 
Dr. SILVA.-Se manifiesta de acuerdo con la iniciativa del doctor Pérez y al efecto nombra para integrar la Comisión de toxemias, distribuídos en todas las clínicas, a los doctores: Fernández y Afanador para la Clínica Primero de Mayo; Pérez para la Clínica "La Magdalena"; Alfonso Ucrós para la Clínica "Santa Lucía"; Capasso para el Hospital de "La Providencia"; Casas para la Clínica "Camero"; Pedraza para la Clínica de "Marly"; Ortiz Gamboa para el Hospital de "San José", y los Jefes de Clínica Obstétrica, Hunda, Pérez, Navas Hernando, Navas Guillermo, Salazar, Riaño y Sanz para el Instituto de Protección Materno-Infantil.

Los doctores Fernández y Afanador, miembros muy activos de esta comisión, empezaron a trabajar desde los días siguientes en la elaboración de un plan de tratamiento de la toxemia. El primer paso dado por estos colegas ha sido efectivo y alentador. Su laboriosidad, tesón e interés científico debe ser imitado por los otros miembros de la comisión de estudios sobre toxemia y deben ser secundados por todos los miembros de la Sociedad.

En la Clínica Primero de Mayo, ellos han conseguido, desde el principio del presente año, el funcionamiento de los siguientes servicios en beneficio de las pacientes toxémicas: local apropiado, reglamento de dietas adecuadas, exámenes de fondo de ojo, instrucciones especiales en Prenatal y Admisión para el pedido y despacho rápidos de los exámenes de laboratorio necesarios; parece que se va a conseguir el entrenamiento de un personal especial para el cuidado de las toxémicas; ellos prestan además en dicha clínica un servicio de consultas para los colegas que allí trabajan, sobre todo lo relacionado para el tratamiento y cuidado de las toxémicas. Finalmente han elaborado una historia especial que han distribuído profusamente en los Hospitales y Clínicas antes citados.

Las funciones de esta comisión se desarrollarán en el curso del presente año y a principios del entrante rendirán un informe a la Sociedad según lo acordado en la sesión del 10 de diciembre de 1953. La Redacción de la Revista ofrece a la Comisión de estudios de toxemia su apoyo amplio con la divulgación de todo lo pertinente al trascendental estudio que ha emprendido, la felicita por la actividad desarrollada en sus funciones y recomienda a todos los miembros de la Sociedad una colaboración desinteresada en estas investigaciones.

El esqueleto de Historia de Toxemia se encuentra en la página siguiente. 


\section{HISTORIA DE TOXEMIA No.}

Clínica: No. de registro:

Dr.

Fecha de ingreso: Nombre:

Edad: Embarazo No. .... Parto No.

Ultima regla:

Peso: ..... Pulso: ...... Edemas: ...... Aumento de peso en el embarazo:

T. A.: ..... Edad del embarazo: .... ¿Feto vivo? .... Presentación:

Antecedentes toxémicos o hipertensivos:

LABORATORIO.-1. Ex. de orina: ¿Albumina? ... Densidad: ... Glucosa: sedimento

2. Azohemia: Uricemia: Glicemia: ¿Proteinemia?

3. Pruebas de función renal:

4. Otros:

5. Examen de fondo de ojo:

TRATAMIENTO MEDICO (anotando la dieta empleada):

¿Aislamiento adecuado? ... ¿Sedación (con drogas)? . . ¿Control de líquidos?... EVOLUCION (en relación con tratamiento empleado):

PARTO.-¿Espontáneo? .... ¿Inducido? .... Tipo de inducción:

Intervención:

Anestesia

Peso del niño al nacer: grs. Talla: ¿Muerto?

DIAGNOSTICO*_Toxemia Aguda._¿Preeclampsia leve? ¿Grave?

¿Eclampsia? No. de ataques:

¿Eclampsia del puerperio? No. de ataques:

Crónica._- ¿Enfermedad hipertensiva?... ¿Enfermedad hipertensiva con toxemia?...

Estado de la madre al salir: T. A.: ¿Abúmina?

Estado del niño al salir: Peso: grs.

* NOTA.-Se considera con pre-eclampsia leve, la enferma con tensión arterial entre 140/90 y $155 / 95$; y grave con tensión $160 / 100$ a más, que persista 24 horas.

OBSERVACIONES: (aquí se anotan datos de evolución clínica, tales como: peso, control de líquidos ingeridos y excretados en 24 horas, exámenes de fondo de ojo, tensión arterial, pulso, resultado de uroanálisis y resumen de terapéutica empleada, con las dosis totales de medicamentos usados).

Si es el caso, se pueden agregar hojas adicionales.

DIAGNOSTICO DEFINITIVO (espacio para ser llenado únicamente por la comisión de estudios de toxemia). 